\title{
ON POLYNOMIALS AND LAGRANGE'S FORM OF THE GENERAL MEAN-VALUE THEOREM
}

\author{
V. RAMASWAMI
}

Suppose that in $(a<x<b)$ (hereafter referred to as $(a, b)$ ), (1) $f(x)$ is defined and has derivatives of the first $n$ orders. Then, from the general mean-value theorem with Lagrange's form of remainder follows the existence of $\theta=\theta(x, h)$, such that

$$
\begin{aligned}
f(x+h)=f(x)+\sum_{r=1}^{n-1} \frac{h^{r}}{r !} f^{(r)}(x)+ & \frac{h^{n}}{n !} f^{(n)}(x+\theta h) \\
& \text { for } a<x<x+h<b .
\end{aligned}
$$

The $\theta$ in (2) is sometimes a uniquely determinate function of $x$ and $h$ in the relevant domain $a<x<x+h<b$ (hereafter referred to as $R$ ), as, for instance, if $f^{(n+1)}(x)$ exists and is not zero in $(a, b)$. If, further, $f^{(n+1)}(x)$ is continuous in $(a, b)$, it is easily seen that

$$
\lim _{h \rightarrow+0} \theta(x, h)=\frac{1}{n+1} \quad \text { in } a<x<b .
$$

It is also possible for $\theta(x, h)$ to be an analytic function, for example,

$$
\theta(x, h)=h^{-1} \log \left(1+\sum_{r=1}^{\infty} \frac{h^{r} \Gamma(n+1)}{\Gamma(n+r+1)}\right),
$$

which happens when $f(x)=e^{x}$.

It would, therefore, seem worth while to determine the types of functions that are or are not possible for $\theta(x, h)$. Inquiry in this direction has led to the results of this paper, namely:

THEOREM 1. If a polynomial $\theta(x, h)$ exists such that (2) is true with $\theta(x, h)$ in place of $\theta$, then $f^{(n+1)}(x)$ exists in $(a, b)$ and either

$$
f^{(n+1)}(x)=0
$$

or

(b) $f^{(n+1)}(x)=a$ constant $\neq 0$ in $(a, b)$, and $\theta(x, h)$ is uniquelydeterminate and equal to $1 /(n+1)$ in $R$.

THEOREM 2. If (2) is true with $\theta(x, h)=c(x)+h^{d} \phi(x, h)$ where

(3) $\phi(x, h)$ is bounded in $R$;

(4) $d$ is a constant greater than 1 ;

Received by the editors January 28, 1948. 
(5) $\partial \theta / \partial x, \partial^{2} \theta / \partial^{2} x$ are continuous in $x$, and $\theta$ is bounded in $R$;

(6) for all sufficiently small $h, 1+h(\partial \theta / \partial x) \neq 0$ in $R$; then, also, (a) and (b) of Theorem 1 are true.

It is significant that, if $\theta$ is uniquely determined by (2) in $R$ and not equal to $1 /(n+1)$, then $\theta=\theta(x, h)$ cannot be equal to a polynomial in $R$ (by Theorem 1 ) or even to an analytic function (by Theorem 2) satisfying

(7) $\lim _{h \rightarrow 0} \partial \theta / \partial h=0$ for every $x$ in $(a, b)$.

[In the following we write $\theta(x, 0)$ for $\lim _{k \rightarrow+0} \theta(x, h)$ and $\theta_{1}(x, 0)$ for $\lim _{h \rightarrow+0}(\theta(x, h)-\theta(x, 0) / h)$ (which limits obviously exist in the contexts of the two theorems), and $\theta_{r s}$ for $\left(\partial^{r+s} / \partial x^{r} \partial h^{s}\right) \theta$, wherever the latter obviously exists.]

Proof of TheOREM 1.

(8) The conditions (5) and (6) are obviously satisfied here and (2) is true by hypothesis.

On account of the consequent boundedness of $\theta$ in $R$, and the continuity of $\theta$ in $x$, follows

(9) $y=x+\theta h$ for every $y$ in $(a, b)$, with any sufficiently small $h$ and a correspondingly chosen $x$ such that $(x, h)$ lies in $R$.

From (8) and (9) follows

(10) $f^{n+1}(x)$ and $f^{n+2}(x)$ exist and are continuous in $(a, b)$.

Now, from the general mean-value theorem follows

$$
\begin{aligned}
f(x+h) & =f(x)+\sum_{r=1}^{n} \frac{h^{r}}{r !} f^{(r)}(x)+\frac{h^{n+1}}{(n+1) !} f^{(n+1)}(x) \\
& +\frac{h^{n+2}}{(n+2) !} f^{(n+2)}\left(x+\theta_{1} h\right), \quad 0<\theta_{1}<1, \quad(x, h) \subset R ;
\end{aligned}
$$

and from (2) and the same theorem applied to $f^{n}(x+\theta h)$ follows

$$
\begin{aligned}
f(x+h) & =f(x)+\sum_{r=1}^{n} \frac{h^{r}}{r !} f^{(r)}(x)+\frac{h^{n+1} \theta}{(n) !} f^{(n+1)}(x) \\
+ & \frac{h^{n+2} \theta^{2}}{n ! 2 !} f^{(n+2)}\left(x+\theta_{2} \theta h\right), \quad 0<\theta_{2}<1,(x, h) \subset R .
\end{aligned}
$$

Subtracting (12) from (11) and making $h \rightarrow+0$ after division by $h^{n+1}$, it follows by (10) that

$$
f^{(n+1)}(x)[1-(n+1) \theta(x, 0)]=0 .
$$

Using (13) in (11) and (12), and making $h \rightarrow+0$ after division of their difference by $h^{n+2}$, it follows, again by (10), 


$$
\begin{aligned}
f^{(n+2)}(x) & {\left[1-\frac{(n+1)(n+2)}{2} \theta^{2}(x, 0)\right] } \\
& -f^{(n+1)}(x)(n+1)(n+2) \theta_{1}(x, 0)=0 .
\end{aligned}
$$

Now, either

(15a) $f^{(n+1)}(x)=0$ everywhere in $(a, b)$;

or or

(15b) $f^{(n+1)}(x) \neq 0$ everywhere in $(a, b)$;

(15c) on account of the continuity (by (10)) of $f^{(n+1)}(x)$ there exists a closed interval $\left(a_{1}, b_{1}\right)$ contained in $(a, b)$ such that $f^{(n+1)}(x) \neq 0$ for $a_{1}<x<b_{1}$, and one at least of $f^{(n+1)}\left(a_{1}\right)$ and $f^{(n+1)}\left(b_{1}\right)$ is zero.

If (15c) were possible, then we should have, by (13) and (14),

$$
\begin{array}{r}
f^{(n+2)}(x) \cdot n / 2(n+1)-f^{(n+1)}(x)(n+1)(n+2) \theta_{1}(x, 0)=0 \\
\text { in }\left(a_{1}<x<b_{1}\right),
\end{array}
$$

and hence $f^{(n+1)}(x)=A \cdot \exp \{\phi(x)\}$ in $a_{1}<x<b_{1}$, where $\phi(x)$ is a polynomial and $A$ is a constant, and making $x \rightarrow a_{1}$ or $b_{1}$ in this, there would follow that $f^{(n+1)}(x)=0$ in $a_{1}<x<b_{1}$, which contradicts (15c). Hence

(16) (15c) is impossible, and $f^{(n+1)}(x)=A \exp \{\phi(x)\}$ in $a<x<b$, where $\phi(x)$ is a polynomial and $A=$ a constant $\neq 0$, if $f^{(n+1)}(x) \neq 0$ for some $x$ in $(a, b)$.

Now differentiating (2) with respect to $x$ and $h$, as is obviously permissible on account of (10), and subtracting, and dividing by $h^{n-1}$, it follows that

$f^{(n)}(x)-f^{(n)}(x+\theta h)=\frac{h}{n} f^{(n+1)}(x+\theta h)\left[\theta-1+h \theta_{01}-h \theta_{10}\right]$ in $R$.

Differentiating this (possible by (10)) with respect to $x$ and using (16) we get

(17) $\exp \{k(x, h)\}=g(x, h)$ in $R$, in case (15b), where $k(x, h)$ $=\phi(x)-\phi(x+\theta h)$ and $k(x, h)$ and $g(x, h)$ are polynomials in $x$ and $h$.

It is now seen by the theory of analytic continuation that (17) is impossible unless $k(x, h)$ is a constant, which again is seen to be zero by keeping $x$ fixed and making $h \rightarrow+0$. Hence

$$
\phi(x)=\phi(x+\theta h)
$$

in $R$.

Now from (2) obviously follows

(19) $f(x)$ is a polynomial of degree not greater than $n$ in $(a, b)$ if $\theta(x, h) \equiv 0$. Also, by continuous variation of $x$ and $h$ in $R$ it follows from (18) that 
(20) $\phi(x)=$ a constant $k$ in $(a, b)$ if $\theta(x, h) \not \equiv 0$, and hence, using (16), follows

(21) $f^{(n+1)}(x)=A e^{k}$ in $(a, b)$ where $A \neq 0$ if $f^{(n+1)}(x) \neq 0$ in $(a, b)$, Now the theorem follows from (10), (15a), (15b), (16), (19) and (21). since, when $f^{(n+1)}(x)=a$ constant $\neq 0, \theta=1 /(n+1)$ and is uniquely determined by (2) in $R$.

Proof of TheOREM 2 . In this case, the statements (8) to (14) follow as above, and $\theta_{1}(x, 0)=0$ since $d>1$. Hence (13) and (14) now become

$$
\begin{array}{cc}
f^{(n+1)}(x)[1-(n+1) c(x)]=0 & \text { in }(a, b), \\
f^{(n+2)}(x)\left[1-\frac{(n+1)(n+2)}{2} c^{2}(x)\right]=0 & \text { in }(a, b) .
\end{array}
$$

Hence either or

(24a) $f^{(n+1)}(x)=0$ every where in $(a, b)$,

(24b) $f^{(n+1)}(x)=c \neq 0$ for some $x$ in $(a, b)$.

Then, (22) and (23) give

(25) $c(x)=1 /(n+1)$ wherever $f^{(n+1)}(x) \neq 0$,

(26) $f^{(n+2)}(x)=0$ wherever $f^{(n+1)}(x) \neq 0$.

The theorem now follows from (10), (24a), (24b), (25) and (26), since, when $f^{(n+1)}(x)=c \neq 0$ in $(a, b), \theta$ in (2) is uniquely determined in $R$.

Note added January 18, 1948. The conclusions (a) and (b) of Theorem 1 are true if (2) holds with $\theta(x, h)$ in place of $\theta$, where

$$
\theta(x, h)=\sum_{r=0}^{m} h^{r} \theta_{r}(x),
$$

and $\theta_{1}(x)$ is a polynomial, $\theta(x, h)$ satisfies (6) and each of the functions $\theta_{\nu}(x)$ satisfies (5). The line of proof is briefly as follows:

The arguments up to and including (16) are the same as above, and the equation in (17) is now true with $K(x, h)=\phi(x)-\phi(x+\theta h)$, and $K(x, h)$ and $g(x, h)$ polynomials in $h$ for fixed $x$. The rest of the argument is the same as before.

The conclusion (20) can also be seen directly as follows: Differentiating (18) with respect to $h$, we have

$$
\phi^{\prime}(x+\theta h)\left(\theta+h \frac{\partial \theta}{\partial h}\right)=0 \text {. }
$$

Making $h \rightarrow 0$ in this and noting that $\theta(x, 0)=1 /(n+1)$ in case $(15 b)$ we have $\phi^{\prime}(x)=0$, and hence $\phi(x)=k$, a constant in $(a, b)$. 